\title{
Molecular Genetics of Substance Abuse Vulnerability: A Current Approach
}

\author{
George R. Uhl, M.D., Ph.D.
}

Which genes contribute to drug abuse vulnerability? How do vulnerability-enhancing alleles work? How are the effects of a specific allelic influenced by other genes and the environment? Identifying the specific gene loci at which allelic variants contribute to human vulnerability to substance abuse is a major goal of the developing field of drug abuse molecular genetics. This work is likely to play an increasingly prominent role in drug abuse research. The feasibility of specific approaches to this problem is now increasingly supported by convergent information from family, twin, and adoption studies of families of drug abusers (see Table 1 for some terms). Results of mouse genetic studies add to our confidence that drug abuse vulnerability genes can be identified. Elucidation of increasingly lengthy lists of candidate genes and rapid development of improving methodologies for approaching complex genetic disorders both enhance the plausibility of identifying such gene variants. The benefits of finding drug abuse vulnerability gene variants are also increasingly appreciated by workers frustrated by our current inability to target prevention and treatment efforts effectively to those whose genetic predispositions leave them most likely to benefit. This review presents a current perspective on the status of approaches to identifying substance abuse vulnerability genes. It reflects material reviewed in two recent meetings as well as background information covered in recent reviews (Uhl et al. 1997;

From the Molecular Neurobiology Branch, Intramural Research Program, National Institute on Drug Abuse, NIH, Box 5180 and Departments of Neurology and Neuroscience, the Johns Hopkins University School of Medicine, Baltimore, MD.

Address correspondence to: Dr. George R. Uhl, P. O. Box 5180, Baltimore, MD 21224, USA.

Received 3 February 1998; revised 11 May 1998; accepted 1 June 1998.
Uhl et al. 1995; Risch and Merikangas 1996; Committee for Problems of Drug Dependence Symposium, Nashville Tenn, July 1997; NIDA Genetics Working Group Meeting, Rockville, MD, October 1997).

\section{GENETIC CONTRIBUTIONS TO HUMAN SUBSTANCE ABUSE VULNERABILITY}

Drug abuse runs in families. Current data from family studies suggest that an abuser's sib has a substantially greater risk of developing a drug problem than if he or she were in the general population. Data reviewed in Croughan (1985) and Uhl et al. (1995) suggest that this risk may increase some five-fold. Few available family studies of drug abusers are well controlled, however. Risks to brothers of affected brothers may be higher than risks for sisters, perhaps reflecting the higher frequency of drug abuse in males than in females (reviewed in Uhl et al. 1995). Adoption studies suggest that some of the familial patterns are genetically mediated. Individuals adopted from substance-abusing parents are more likely to become substance abusers than the others raised in identical environmentals (Cadoret et al. 1986, 1995).

Perhaps most interesting are the twin studies. Studies of thousands of monozygotic and dizygotic twin pairs now reveal strong evidence for genetic contributions, averaging more than $40 \%$, to heritability of two different drug abuse phenotypes (Grove et al. 1990; Pickens et al. 1991; Goldberg et al. 1993; Tsuang et al. 1996; Woodward et al. 1996; M. Tsuange personal communication, 1997; K. Kendler, personal communication 1997). Genetic infuences contribute to the likelihood of an individual's displaying the first phenotype, psychiatric diagnostic and statistical manual (DSM) criteria 
Table 1. Some Terms

Adoption studies in drug abuse: Studies in which the prevalence of drug abuse is compared in adoptees, their biological parents, their adoptive parents, and their adoptive sibs. Increased risk (compared to adoptive sibs) allows assessment of genetic influences. Decreased risk (compared to their parents) can even allow assessment of the magnitude of environmental influences.

Association studies: As used here, compare gene marker frequencies in unrelated individuals with a disorder to those in control individuals without the disorder.

Genetic heterogeneity: Several different genes (nonoverlapping) or several different groups of gene variants can cause the same disorder (phenotype). Thus, the gene variants that cause substance abuse in one individual could be different from those in another individual with a similar drug abuse disorder.

Genetic isolates: Populations that have been derived from so few founders and maintained their genetic isolation so that a relatively few founder chromosomes are heavily represented. Relatively fewer recombination events separate the chromosomes of one member of the population from those of another.

Genome scanning methods: Studies seeking DNA markers that are inherited by individuals along with a disorder at greater than chance levels. In practice, many DNA markers located up and down each chromosome are studied.

Heritability: The extent to which a trait or disease can be inherited, the influence of genetic mechanisms in a disorder.

$L O D$ score: $\log$ of the odds ratio methods used for standard Mendelizing genetic disorders, generally used with assumed inheritance as single gene disorders.

Mendelizing disorder: Disorder inherited as a single gene disorder, producing characteristic patterns of inheritance. $50 \%$ of offspring will manifest a classical Mendelizing autosomal dominant trait, for example, if a single parent is affected.

Phencopies: Disorders that appear similar in clinical manifestations, but have different underlying mechanisms (genetic or environmental).

Proband: Initial individual in a family, usually the initial individual identified with a disorder.

Polymorphism: As used here, represents a specific DNA sequence difference between some humans and other humans. These sequence differences can serve as markers, or can have functional consequences for the gene that contains the polymorphism.

QTL: Quantitative trait locus analyses find influences of specific chromosomal regions in producing the mouse strain differences in quantitative traits, such as drug consumption.

Segregation analysis: Studies of the ways in which traits move through families. Traits that have the same genetic bases should move through families together; whereas, those that depend upon different alleles could be inherited separately and, thus, move through families separately.

Twin studies in drug abuse: Studies in which the prevalence of drug abuse is compared in monozygotic twins that are genetically identical with prevalence in dizygotic twins that share $50 \%$ identical genes, on average. Greater mono- than dizygotic twin similarity (concordance) generally indicates genetic influence. for substance abuse or dependence. They also contribute, perhaps even more strongly, to the likelihood of a second phenotype: high-quality/high-frequency substance use (Goldberg et al. 1993; Tsuang et al. 1996; M. Tsuang personal communication, 1997). In two independent factor analyses of twin datasets, a factor representing an underlying genetic vulnerability can explain much of the variance in use of several different abused substances (C. Pearson personal communication 1997; M. Tsuang personal communication 1997). However, heroin use by those individuals who sampled it during the Vietnam era, largely intravenous, may display genetic contributions less shared with those predisposing to other substances (M. Tsuang, personal communication 1997).

Epidemiological surveys indicate elevated frequencies of drug abuse among individuals who are also diagnosed with antisocial personality disorder, depression, alcoholism, and perhaps adult residua of attention deficit hyperactivity or conduct disorders, disorders that themselves display only modest overlaps (Regier et al. 1990; Rounsaville et al. 1991; Wilens et al. 1997). Although the alleles that contribute to drug abuse may not be entirely identical to those that help produce these co-morbid disorders, several could be contributors. Each candidate locus identified for these disorders, thus, becomes an attractive candidate gene locus for contributions to drug abuse vulnerability. The co-morbidities could also provide potential cleavage planes to help to elucidate genetic heterogeneity among possible subtypes of drug abusers (Rounsaville et al. 1991; Wilens et al. 1997). However, studies of families in which multiple generations display substance abuse problems reveal that alcohol abuse can segregate in these pedigrees differently from drug abuse (Hill et al. 1977; Merikangas et al. personal communication 1997). The gene variants underlying alcoholism, or perhaps any of these common comorbidities, may include those not shared with substance abuse, as well as others that may be shared with substance abuse.

\section{STUDIES IN ANIMAL MODELS}

Mice are a major model system for behavioral genetic studies. Mice can display conditioned place preferences for and self-administration of virtually all of the substances abused by humans (Belknap and O'Toole 1991; Belknap and Crabbe 1992; Crabbe et al. 1994). The murine responses in each of the major current animal models for drug abuse, or at least the rewarding properties of drugs, can be substantially modulated by variation at single and at multiple gene loci. Quantitative trait locus studies have implicated variation at several interesting candidate chromosomal regions; for example the region 
that contains the $\mu$ opiate receptor locus, in drug selfadministration (Berettini et al. 1994). Effects at single gene loci can also be studied using transgenic mice. These studies now convincingly demonstrate major effects on drug reward from alterations at dozens of single gene loci (e.g. Takahashi et al. 1997; Sora et al. 1997; Maldonado et al. 1997). Often heterozygous knockout transgenic mice display variations in gene expression of roughly the same magnitudes as those defined for human interindividual differences (see Sora et al. 1997 compared to Pfeiffer et al. 1982; Frost et al. 1995). When mouse expression differences of the sizes found in humans lead to differences in drug responses, these models may have significant direct consequences for thinking about human drug abuse disorders.

Mice also represent a plausible means to help define which pieces of a large chromosomal segment identified in a genome scanning study could contain functionally important polymorphisms. This might be especially true for genes whose function depends upon gene dose. Transgenic mice that overexpress varying genomic segments that contain such genes could be subjected to behavioral screens to assess which segments might alter behaviors in model drug reward assays. Smaller segments that induce altered drug responses could be more readily scanned to identify expressed genes and the products identified. Conceivably, such approaches could enhance the efficiencies of attempts to localize important sequences, as compared to unaided human genetic approaches.

\section{MODES FOR GENETIC CONTRIBUTIONS TO DRUG ABUSE VULNERABILITIES AND THEIR IMPLICATIONS}

Several features of drug abuse provide difficulties for traditional pedigree studies that can provide reliable data about mode of inheritance for many disorders. Because drug fashion and availability can differ dramatically from one year to another, different human cohorts have been exposed to substantially different drug classes, preferred routes of administration, and so forth (Gynther et al. 1995). Drug abusers are often members of disorganized families. In this setting, careful segregation analyses that might provide evidence for the presence or absence of major gene effects in drug abuse vulnerability are difficult. The high frequencies of drug abuse problems in the U.S. population do suggest that it is unlikely that rare allelic variants make major contributions to this disorder (SAMHSA 1995). The common but distinct co-morbidities, noted above, also provide a line of evidence for likely genetic heterogeneity.

Approaches to identifying substance abuse vulnerability genes could take two parallel tracks, in the pres- ence of suggestive data for genetic heterogeneity and the absence of data that convincingly document major gene effects (see Table 2). They could consider the possibility that some loci with moderate size effects might exist and also the possibility that most of the locus-specific effects on over-all vulnerability might be small. Recent modeling and power calculations suggest that genome scanning approaches with substantial sample sizes would have a reasonable likelihood of detecting a major gene effect that commonly caused the disorder. A locus contributing as little as $10 \%$ of the over-all variance, for example, could be detected with reasonably good power by genome-scanning approaches using markers of reasonable density and 2,250 affected sib pairs (Risch and Merikangas 1996; Risch 1990; A. Wilson personal communication). However, such approaches are unlikely to detect alleles that contribute less of the variance unless enormous samples are studied or very closely spaced markers are employed (Collins et al. 1997). Candidate gene approaches allow development and use of the most closely spaced markers and provide hypothesis-driven assessments that gain substantial statistical power (Risch and Merikangas 1996; Uhl et al. 1997). Thus, both genome scanning and candidate gene approaches can find good utility in drug abuse molecular genetics.

What sorts of family material would be most useful to collect? The genetic approaches that allow comparisons between individuals of roughly the same age

Table 2. Candidate Gene and Locus Exclusion Approaches for Drug Abuse and Dependence

Sources of variation:

Levels of expression. Possibly more common variation underlying common complex disorders, but more difficult to elucidate

Protein coding. Possibly less common variation underlying common complex disorders, but more readily elucidated

Exclusion of roles for variants at a specific gene locus are excluded with increasing confidence as:

Aspects of gene structure identified (e.g. coding exons) are usually identified first

Polymorphic markers are then often defined in or near coding exons.

Individual differences in protein or expression levels are identified along with possible functional polymorphisms.

Associations of the initial polymorphisms with drug abuse are often sought.

Aspects of regulatory regions often defined later

Distributions of polymorphisms in different ethnic/racial subpopulations are identified

SSCP and exon sequencing to search for expressed sequence variants

Increasing coverage of locus coding and regulatory regions with markers in good linkage disequilibrium with each other

Tests of association between drug abuse and locus-spanning markers allows exclusion of the locus with increasing confidence. 
ranges (e.g. haplotype relative risk, transmission disequilibrium, and affected sib pair approaches) can be of especial value when secular trends in drug fashion and availability can dramatically alter a gene variant's expressivity (e.g. Gynther et al. 1995). Studies of research volunteers that come from the same sorts of clinical populations in which twin studies have already documented good heritabilities provides added assurance. Otherwise, power could be diluted by inclusion in genetic analyses of large numbers of individuals with environmentally determined phenocopies.

Studies of substance abuse in genetic isolates or in pedigrees in which it displays an apparent Mendelizing pattern provide alternative approaches. Studies in genetically isolated populations can reduce the genetic variation examined and add to the informativeness of polymorphic genetic markers (Collins et al. 1997). However, the substance use among the currently available genetic isolates often used for studies of other disorders may not sufficiently resemble those in the general U.S. population to predict generalizable results with confidence. Although occasional pedigrees with apparent Mendelian patterns of inheritance can sometimes be obtained for drug abuse, these pedigrees are uncommon. The apparent low likelihood that a single gene causes drug abuse also reduces enthusiasm for log of the odds (lod) score approaches to such rare families.

\section{IMPROVED GENOMIC INFORMATION TO AID IDENTIFICATION OF DRUG VULNERABILITY CANDIDATE GENES}

Identifying drug abuse vulnerability genes that produce modest differences in vulnerability will require the maximal possible sensitivity of the molecular genetic approaches used. The sensitivity and specificity with which genetic approaches can identify gene variants that make such modest contributions to vulnerability depends upon testing markers as close as possible to the actual site of the allelic gene variant (Risch and Merikangas 1996; Collins et al. 1997). Studying many markers up and down the genome, including the single nucleotide polymorphisms (SNPs) that occur every few hundred base pairs, is one approach. Knowledge about the neurobiology of drug abuse supplements this approach. It focuses attention on specific candidate genes' protein coding or regulatory region polymorphisms as sources for functional differences in the structures or expression.

Candidate genes for drug abuse vulnerability can be identified in several ways. Drug "receptor" sites of acute drug action, genes that can be regulated by drug administration in experimental animals or in humans, genes expressed in brain circuits and biochemical pathways important for drug actions, genes lying in ge- nomic regions identified from murine quantitative trait locus (QTL) approaches, and genes that are implicated in major substance abuse co-morbidities provide some of the best approaches (Uhl et al. 1995; Institute of Medicine, Committee on Opportunities in Drug Abuse Research 1996). The dopamine and synaptic vesicular monoamine transporters (DAT and VMAT2), for example, provide strong candidate genes, because they are expressed in dopaminergic neurons centrally implicated in reward and the neurons on which cocaine and amphetamine have a direct impact (Uhl et al. 1998). Genome scanning approaches may identify genes in the regions of chromosomal markers that are preferentially coinherited with commonly co-morbid conditions or with substance abuse vulnerabilities. Such loci are strong potential candidates as well.

Candidate gene approaches to drug abuse molecular genetics require thinking about several genomic features of the candidate genes. What are their structural and regulatory regions? What variation occurs at these loci? Variations that provide markers that do not alter gene functions, those that alter gene regulatory function, and those that change protein-coding sequences are all interesting to study. How do these gene variants distribute through families? Through the population? Candidate gene approaches also depend upon the power of drug abuse neurobiology and genetics to inform us about which genes are the best candidates and how a given gene variant might act to alter drug abuse vulnerabilities.

Thinking about drug abuse can draw from studies of other complex disorders, those in which several genes and environmental factors are likely to play roles. These power calculations suggest that candidate gene approaches represent the most powerful means of detecting gene variants that make small contributions to individual differences in drug abuse vulnerabilities (Risch and Merikangas 1996; Uhl et al. 1997). Thus, maximizing the precision of such candidate gene approaches for drug abuse is increasingly important. As one approach to the initial problem of defining how many candidate genes might currently be identified, we have recently surveyed 1,000 human genes lying on chromosomes 1 to 3 and assigned them to the categories listed in Table 3 (GRU and D. Vandenbergh unpublished observations 1997). When two experience molecular neurobiologists rated database gene entries according to these criteria, 1 to $2 \%$ of the genes were identified as the strongest type 1 candidates. About $5 \%$ were type 2, about $10 \%$ were type 3 , and the rest were type 4 . These data provide an initial rough estimate of the work that might be entailed in examining the best currently available candidate gene loci. Carefully examining even the 1 to $2 \%$ of the ca. 80,000-100,000 human genes that may represent the best current a priori candidate genes for drug abuse vulnerability provides a major task for drug abuse gene 
Table 3. Candidate Genes and Chromosomal Regions for Drug Abuse and Dependence

\begin{tabular}{l} 
Sources: \\
Drug receptor genes \\
Drug regulated genes \\
Genes expressed in reward and memory circuits \\
Chromosomal regions implicated in comorbid disorders \\
Chromosomal regions syntenic with murine QTL loci \\
Results of applying a proposed categorization of candidate \\
genes for drug abuse and dependence to 1,000 genes on \\
chromosomes 1 to 3 (GRU and D. Vandenbergh, \\
unpublished observations 1997) \\
Type 1: Strongly implicated in actions of abused substances or \\
in substance abuse genetic models Type 1 result: 1 to $1.5 \%$ \\
Type 2: Present in fashions possibly involved in actions of \\
abused substances or in substance abuse genetic models \\
Type 2 result: 3 to $5 \%$ \\
Type 3: More likely than other genes with present knowledge \\
Type 3 result: $10 \%$ \\
Type $4:$ No more likely than any other gene with present \\
knowledge Type 4 result: rest \\
\hline
\end{tabular}

discovery efforts. Careful exclusion of contributions of variation at these loci to drug abuse vulnerability will require major effort.

How can we have increasing confidence that we can exclude a chromosomal locus with current genome scanning and candidate gene approaches? For Mendelizing disorders, assessment of the extent of coinheritance of a single highly informative candidate gene locus marker along with the trait can allow straightforward exclusion of hypothesis that genetic variation at the locus causes the disease with high statistical certainty (Ott 1991). It would be desirable to approach this degree of certainty for drug abuse. Increasing confidence in our abilities to exclude candidate loci requires increasing knowledge about the locus, about the genetic variability that the locus displays in different ethnic and disease populations, and about the diseases being approached. Increasing knowledge about coding sequences and the sources of variation in these sequences can be complemented by studies of gene promoter/enhancer sequences and the sources of variation in these regulatory regions. To the extent that roles of allelic variants in different ethnic, racial, or disease populations are postulated, examination of gene marker distributions in these subpopulations should also be sought. This combined knowledge can allow increasing confidence that contributions of the variations noted to the etiology of these disorders can be established. Ultimately, more and more candidate gene loci can be excluded as making significant contributions to these disorders with increasing confidence. It is hoped that a few will also resist our attempts at exclusion.

\section{SUMMARY: INITIAL APPROACHES TO IDENTIFYING HUMAN SUBSTANCE ABUSE VULNERABILITY GENES}

Initial parallel efforts for identifying human substance abuse vulnerability genes can proceed on several tracks to maximize the chances that gene identification efforts will ultimately lead to useful successes.

Clinical studies must identify small nuclear families for affected sib pair of other related methods for genome scanning and candidate allele approaches. Power calculations do depend upon the mode of inheritance; for example, dominant versus recessive (Risch 1990). Studies of sibships in which: (1) sibs of the same gender and similar ages are discordant for drug abuse phenotypes; and (2) both parents' genotypes can be determined provides maximal power under most modes of inheritance. In our initial studies in a research volunteer population, such families represent a minority of those that can be determined when a proband volunteers for a research study. Characterizing phenotypes based on (1) quantity/frequency; and (2) DSM diagnoses, the two phenotypes for which most twin data suggest substantial heritability, seems to be a good initial bet. However, improving the precision of these diagnostic approaches and their genetic loading through more focused twin and family studies, including segregation studies, is also likely to be helpful (e.g. see Johnson et al. 1996; Pickens et al. 1995). Careful attention to the ethical issues inherent in approaching families about the genetics of vulnerability to an illegal behavior is mandatory. One such approach, allowing the proband to choose the means of contacting other family members, is indicated in Table 4.

Table 4. Consent for Approaches to Members of DrugAbusing Families

Consent to contact relatives: Our ability to find a relationship between specific genes and substance abuse is greatly increased if we can study selected blood relatives (that is, not step or adopted) of people who participate in this study. Therefore, we are requesting your help in this work.

May we have your permission to have our third party contractor contact any of your blood relatives to ask them to participate in this study? They will not be given any information about you except that you gave us permission to contact them. We will contact only those family members that you have specified on a separate sheet.

\section{Intial-}

If you are not comfortable with our contacting any relative, would you be willing to contact your brothers, sisters, and biological parents to ask them to participate in this research project?

\section{Initial-}

If you do not want any contact with your relatives we want to record this, but you can still participate in this study.

Initial- 
Samples of unrelated individuals are also useful for classical association studies. As methods for pooled genotyping become more widely validated (e.g., Barcellos et al. 1997; Syvanen et al. 1997), these individuals' samples will become even more valuable.

Improving understanding of the relevant portions of the genome is a second class of preliminary step. Better definition of possibly important candidate gene loci and their human variants improves our ability to include or exclude contributions of this variation to drug abuse phenotypes. Data from transgenic mouse studies now indicate that modest effects of candidate gene variants on human behaviors could be identified, if the correct behaviors are appropriately assessed.

Improving the utility of animal models provides a third source of useful preliminary work. Better models will be likely to allow more rapid assessment of the roles of genes lying in a large interval, especially if their overexpression leads to a dominant phenotype.

As these preliminary steps are being completed, genome scanning can be undertaken in members of the pedigrees with markers spaced as closely as possible across the genome. Positive findings for markers lying in a chromosomal region can then be replicated in other clinical samples, including association samples. Interesting genes lying in the candidate regions can be studied. Mice overexpressing fragments of the interesting region can be evaluated in appropriate behavioral protocols. Searches for sources of variation can make candidate gene loci as informative as possible, so that studies in association and pedigree populations can proceed. These studies of the genes can be supplemented with those examining the candidate gene products directly, for example by in vivo imaging.

What could the results of such searches be? Ability to predict enhanced vulnerabilities to substance abuse should be available from relatively inexpensive genetic screening testing, such as that which should result from use of DNA chips or microarrays. Such information should help focus prevention resources on those individuals most likely to benefit from them. It could also provide life problems, including a basis for discrimination in insurability or job finding. The ability to match treatments with the substance abusers most likely to benefit from them would help to triage still-too-scarce treatment funds to those who most need them. Identifying gene variants that enhance vulnerability, even in subgroups of abusers, could enrich our understanding of the mechanisms that underlie addiction in all individuals. Finally, better comprehension of genetic predisposing factors provides significantly enhanced power to detect environmental factors in drug abuse, because the genetic "noise" can be eliminated from studies of environmental influences. Clearly, significant discussions about the ethical concerns that increasing genetic information provides should proceed in parallel with elucidation of the genes involved. However, with care, it is possible that some of the most significant advances in drug abuse research with applications for prevention and treatment will arise from carefully planned and executed programs of genetic research into the difficult problems that drug abuse presents for individuals and for society.

\section{ACKNOWLEDGMENTS}

The author gratefully acknowledges the contributions to these ideas and work in his laboratory by a number of exceptional students and fellows, thoughtful comments on the manuscript by D. Vandenbergh, Qui-Rong Liu, and Z. Lin, and support from the NIDA-IRP. This work represents a personal opinion, but is informed by many helpful conversations with Jonathan Pollock and other members of the genetics Working Group, NIDA.

\section{REFERENCES}

Barcellos LF, Klitz W, Field LL, Tobias R, Bowcock AM, Wilson R, Nelson MP, Nagatomi J, Thomson G (1997): Association mapping of disease loci by use of a pooled DNA genomic screen. Am J Hum Genetics 61:734-747

Belknap JK, O’Toole LA (1991): Studies of genetic differences in response to opioid drugs. In Harris RA, Crabbe JC (eds), The Genetic Basis of Alcohol and Drug Actions. New York, Plenum Press, pp 225-252

Belknap JK, Crabbe JC (1992); Chromosome mapping of gene loci affecting morphine and amphetamine responses in the BXD recombinant inbred mice. In Kalivas PW, Samson $\mathrm{HH}$ (eds), The neurobiology of drug and alcohol addiction. New York, The New York Academy of Sciences, pp 311-323

Berettini WH, Ferraro TN, Alexander RC, Buchberg AM, Vogel WH (1994): quantitative trait loci mapping in three loci controlling morphine preference using inbred mouse strains. Nature Genet 7:54-58

Cadoret RJ, O'Gorman T, Troughton E, Heywood E (1986): An adoption study of genetic and environmental factors in drug abuse. Arch Gen Psych 43:1131-1136

Cadoret RJ, Yates E, Troughton E, Woodworth G, Stewart MA (1995): Adoption study demonstrating two genetic pathways to drug abuse. Arch Gen Psychaitr 52:42-52

Collins FS, Guyer MS, Chakravarti A (1997): Variations on a theme: Cataloging human DNA sequence variation. Science 278:1580-1581

Crabbe JC, Belknap JK, Buck KJ (1994): Genetic animal models of alcohol and drug abuse. Science 264:1715-1723

Croughan JL (1985): The contribution of family studies to understanding drug abuse. In Robbins L (ed), Studying Drug Abuse. New Brunswick, NJ, Rutgers University Press

Frost JJ, Douglass KH, Mayberg HS, Dannals RF, Links JM, Wilson AA, Ravert HT, Croier WC, Wagner HN (1995): 
Multicompartmental analysis of $\left[{ }^{11} \mathrm{C}\right]$-carfentanil binding to opiate receptors in humans measured by positron emission tomography. J Cereb Blood Flow Metab 9:398409

Goldberg J, Lyons MJ, Eisen SA, True WR, Tsuang, M (1993): Genetic influence on drug use: A preliminary analysis of 2,674 Vietnam era veteran twins. Behav Genet 23:552

Grove WM, Eckert ED, Heston L, Bouchard TJ, Segal N, Lykken DT (1990): Heritability of substance abuse and antisocial behavior: A study of monozygotic twins reared apart. Biol Psychaitr 27:1293-1304

Gynther LM, Carey G, Gottesman I, Vogler GP (1995): A twin study of nonalcohol substance abuse. Psychaitr Res $56: 213-220$

Hill SY, Cloninger CR, Ayre FR (1977): Independent familial transmission of alcoholism and opiate abuse. Alcohol Clin Exper Res 1:335-342

Institute of Medicine, Committee on Opportunities in Drug Abuse Research (1996): Pathways of Addiction. Washington, DC, National Academy Press

Johnson EO, van den Bree MB, Uhl GR, Pickens RW (1996): Indicators of genetic and environmental influences in drug-abusing individuals. Drug Alcohol Depend 41:17-23

Maldonado R, Salardi A, Valverde O, Samad TA, Roques BP, Borelli E (1997): Absence of opiate rewarding effects in mice lacking dopamine $\mathrm{D}_{2}$ receptors. Nature 388:586589

Ott J (1991): Analysis of Human Genetic Linkage. Baltimore: Johns Hopkins University Press

Pfeiffer A, Pasi A, Mehraein P, Herz A (1982): Opiate receptor binding sites in human brain. Brain Res 248:887-896

Pickens RW, Svikis DS, McGue M, LaBuda MC (1995): Common genetic mechanisms in alcohol, drug, and mental disorder co-morbidity. Drug Alcohol Depend 39:129_ 138

Pickens RW, Svikis DS, McGue M, Lykken DT, Heston LL, Clayton PJ (1991): Heterogeneity in the inheritance of alcoholism: A study of male and female twins. Arch Gen Psych 48:19-28

Regier DA, Farmer ME, Rae DS, Locke BZ, Keith SJ, Judd LL, Goodwin FK (1990): Comorbidity of mental disorders with alcohol and other drug abuse. JAMA 264:25112518

Risch N (1990): Linkage strategies for genetically complex traits. Am J Hum Genetics 46:222-232
Risch N, Merikangas K (1996): The future of genetic studies of complex human diseases. Science 273:1516-1517

Rounsaville BJ, Kosten TR, Weissman MM, Prusoff B, Pauls DL, Anton SF, Merikangas K (1991): Psychiatric disorders in relatives of probands with opiate addiction. Arch Gen Psych 48:33-42

Substance Abuse and Mental Health Services Administration (SAMHSA) (1995): National Household Survey on Drug Abuse: Population Estimates. Washington, DC, U.S. Dept. of Health and Human Services

Sora I, Takahashi N, Funada M, et al. (1997): Opiate receptor knockout mice define mu receptor roles in endogenous nociceptive responses and morphine-induced analgesia. Proc Nat Acad Sci 94:1544-1549

Syvanen AC, Tilgmann C, Rinne J, Ulmanen I (1997): Genetic polymorphism of catechol-O-methyltransferase (COMT): correlation of genotype with individual variation of S-COMT activity and comparison of the allele frequencies in the normal population and parkinsonian patients in Finland. Pharmacogenetics 7:65-71

Takahashi N, Miner LM, Sora I, et al. (1997): VMAT2 knockout mice: Heterozygotes display reduced amphetamineconditioned reward, enhanced amphetamine locomotion, and enhanced MPTP toxicity. Proc Nat Acad Sci 94:9938-9943

Tsuang MT, Lyons MJ, Eisen SA, Goldberg J, True W, Li N, Meyer JM, Toomey R, Faraone SV, Eaves L (1996): Genetic influences on DSM-III-R drug abuse and dependence: A study of 3,372 twin pairs. Am J Med Genet 67:473-477

Uhl GR, Elmer GI, Labuda MC, Pickens RW (1995): Genetic influences in drug abuse. In Bloom FE, Kupfer DJ (eds), Psychopharmacology: The Fourth Generation of Progress. New York, Raven Press, pp 1793-1806

Uhl GR, Gold LH, Risch N (1997): Genetic analyses of complex behavioral disorders. Proc Nat Acad Sci 94:27852786

Uhl GR, Vandenbergh DJ, Rodriguez AL, Miner L, Takayashi N (1998): Dopaminergic genes and substance abuse. Adv Pharmacology 42:1024-1032

Wilens TE, Biederman J, Mick E, Faraone SV, Spencer T (1997): Attention deficit hyperactivity disorder (ADHD) is associated with early onset substance use disorders. J Nerv Ment Dis 185:475-482

Woodward CE, Maes HH, Silberg JL, Meyer JM, Eaves LJ (1996): Tobacco, alcohol, and drug use in 8-16-year-oldtwins. NIDA Res Monogr 162:309 